\title{
LOGISTIC FUNCTION AS A TOOL OF PLANNING
}

\author{
Grzegorz RZĄDKOWSKI*, Iwona GŁAŻEWSKA**, Katarzyna SAWIŃSKA*** \\ Warsaw University of Technology, Faculty of Management, Warsaw, Poland \\ *e-mail: g.rzadkowski@wz.pw.edu.pl \\ **e-mail: i.glazewska@wz.pw.edu.pl \\ ***e-mail: katarzyna-s@o2.pl
}

\begin{abstract}
In the present paper, we propose a new approach to investigate the logistic function, which is commonly used in mathematical models in economics and management. The approach is based on indicating in a given time series, having a logistic trend, some characteristic points corresponding to zeroes of successive derivatives of the logistic function. We also give examples of application of this method.
\end{abstract}

Keywords: Logistic equation, logistic function, time series, Eulerian numbers, Riccati's differential equation, mathematical models.

1

\section{Introduction}

The logistic equation is defined as

$$
u^{\prime}(t)=c_{1} u\left(u_{\max }-u\right), \quad u(0)=u_{0}>0,
$$

where:

$t$ is time,

$u=u(t)$ is an unknown function,

$c_{1}, u_{\max }$ are constants.

The constant $u_{\max }$ is called the saturation level. The integral curve $u=u(t)$ of the equation (1), fulfilling the condition $0<u(t)<u_{\max }$ is known as the logistic function.

Many of economic phenomena, also related to the management follows equation (1) (see papers [3, 4, 5, 9, 10, and 11]).

A phenomenon described by equation (1) and function $u(t)$ has an important property that the rate of growth $u^{\prime}(t)$ is proportional to the level already achieved, i.e. $u(t)$.

On the other hand, if $u(t)$ is sufficiently large, then the factor $\left(u_{\max }-u\right)$ is more and more significant and its influence inhibits further growth of the function $u(t)$.

Mathematically equation (1) is the first order ordinary differential equation, which is easily solved by the method of the separation of variables.

The main idea of the present paper is to look, among the data of a given time series, for some characteristic points, which correspond to zeroes of derivatives of the logistic function. One of these points is clearly the point corresponding to the inflection point (i.e. the zero of $u^{\prime \prime}$ ) of the logistic curve at which, as is well known, the logistic function takes the value $u_{\max } / 2$. For a sufficiently long time series, the point corresponding to the inflection point is easy to locate, even from the graph. If the data were collected for the time points spaced equally, then, instead of estimating the values of the first derivative, it is sufficient to calculate successive differences and seek the maximum for them.

What we can do, however, when the time series is not long enough, and we expect that the investigated phenomenon follows the logistic curve? When the phenomenon is on early stage of growth and the data is available only in a relatively short time interval? Statistical methods for estimating the parameters of the logistic function based, for example, on the method of the nonlinear least squares may be unreliable, because functions having significantly different values of the saturation level may produce only slightly differing error values. A way to explanation of the situation seems in seeking, in the time series, points corresponding to zeroes of successive derivatives of the logistic function. For equally spaced time points, this is equivalent to calculating successive differences. For example, as we will see in Section 3, the zero of the third derivative $u^{\prime \prime \prime}$ (i.e., the extreme (maximum) of the second derivative $u^{\prime \prime}$ ) occurs at the point where the value of the logistic function is approximately $0.211 u_{\max }$. 


\section{Logistic equation and logistic function}

We rewrite the logistic equation (1) into the following, more convenient form, where the constant $c_{1}$, for computational reasons, is written as $c_{1}=c / u_{\max }$ :

$$
u^{\prime}(t)=\frac{c}{u_{\max }} u\left(u_{\max }-u\right), \quad u(0)=u_{0} .
$$

After solving equation (2), we get the logistic function in the following form

$$
u(t)=\frac{u_{\max }}{1+a e^{-c t}},
$$

where constant $a$ appears in the integration process and is connected with the initial condition $u(0)=u_{0}=\frac{u_{\max }}{1+a}$, therefore $a=\frac{u_{\max }-u_{0}}{u_{0}}$.

Fig. 1 shows the graph of an exemplary logistic function with parameters $u_{\max }=7, a=17$ and $c=1.5$.

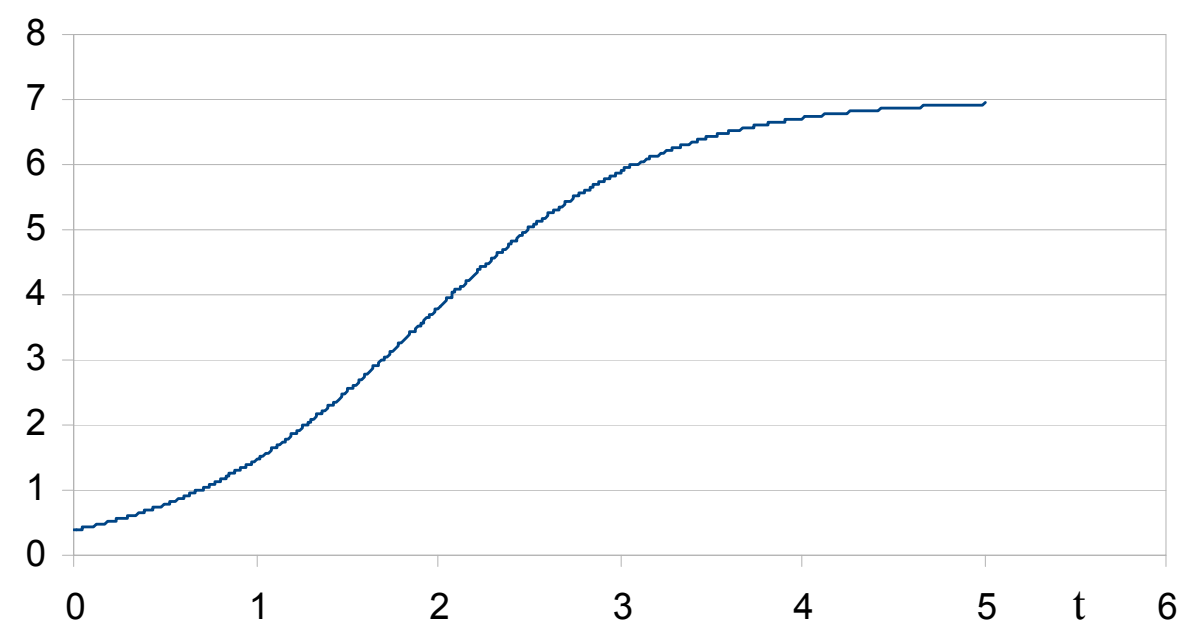

Figure 1. Logistic function with parameters: $u_{\max }=7, a=17$ and $c=1.5$

In order to understand the further reasoning, we have to introduce the so-called Eulerian numbers (see for instance Graham et al. [2]). Let $\left\{a_{1}, a_{2}, \ldots, a_{n}\right\}$ be a permutation of the set $\{1,2, \ldots, n\}$. Then $\left\{a_{j}, a_{j+1}\right\}$ is an ascent of the permutation, if $a_{j}<a_{j+1}$. The Eulerian number denoted by $\left\langle\begin{array}{l}n \\ k\end{array}\right\rangle$ is defined as the number of permutations of the set $\{1,2, \ldots, n\}$ having $k$ ascents $(k=0,1,2, \ldots, n-1)$. For example, for $n=3$ the identity permutation $\{1,2,3\}$ has two ascents, namely $\{1,2\}$ and $\{2,3\}$, and $\{3,2,1\}$ has no ascents. Each of the other four permutations of the set has exactly one ascent. Thus,

$$
\left\langle\begin{array}{l}
3 \\
0
\end{array}\right\rangle=1,\left\langle\begin{array}{l}
3 \\
1
\end{array}\right\rangle=4 \text { and }\left\langle\begin{array}{l}
3 \\
2
\end{array}\right\rangle=1 .
$$

The first few Eulerian numbers are given in the Table 1. It is well known that Eulerian numbers satisfy the following relations:

$$
\begin{aligned}
& \left\langle\begin{array}{l}
n \\
k
\end{array}\right\rangle=\left\langle\begin{array}{c}
n \\
n-k-1
\end{array}\right), \\
& \left\langle\begin{array}{c}
n+1 \\
k
\end{array}\right\rangle=(k+1)\left\langle\begin{array}{l}
n \\
k
\end{array}\right\rangle+(n-k+1)\left\langle\begin{array}{c}
n \\
k-1
\end{array}\right\rangle,
\end{aligned}
$$$$
\left\langle\begin{array}{l}
n \\
k
\end{array}\right\rangle=\sum_{j=0}^{k}(-1)^{j}\left(\begin{array}{c}
n+1 \\
j
\end{array}\right)(k-j+1)^{n} .
$$ 
Table 1. Eulerian numbers

\begin{tabular}{|c|c|c|c|c|c|c|c|c|}
\hline$n$ & $\left\langle\begin{array}{l}n \\
0\end{array}\right\rangle$ & $\left\langle\begin{array}{l}n \\
1\end{array}\right\rangle$ & $\left\langle\begin{array}{l}n \\
2\end{array}\right\rangle$ & $\left\langle\begin{array}{l}n \\
3\end{array}\right\rangle$ & $\left\langle\begin{array}{l}n \\
4\end{array}\right\rangle$ & $\left\langle\begin{array}{l}n \\
5\end{array}\right\rangle$ & $\left|\begin{array}{l}n \\
6\end{array}\right\rangle$ & $\left\langle\begin{array}{l}n \\
7\end{array}\right\rangle$ \\
\hline 0 & 1 & & & & & & & \\
\hline 1 & 1 & 0 & & & & & & \\
\hline 2 & 1 & 1 & 0 & & & & & \\
\hline 3 & 1 & 4 & 1 & 0 & & & & \\
\hline 4 & 1 & 11 & 11 & 1 & 0 & & & \\
\hline 5 & 1 & 26 & 66 & 26 & 1 & 0 & & \\
\hline 6 & 1 & 57 & 302 & 302 & 57 & 1 & 0 & \\
\hline 7 & 1 & 120 & 1191 & 2416 & 1191 & 120 & 1 & 0 \\
\hline
\end{tabular}

Equation (2) is a particular case of the following Riccati differential equation with constant coefficients

$$
u^{\prime}(t)=r\left(u-u_{1}\right)\left(u-u_{2}\right) .
$$

On the right hand side of equation (4) is a quadratic function of the variable $u$ with coefficient $r$ of $u^{2}$ and having roots $u_{1}, u_{2}$. The constants $r \neq 0, u_{1}, u_{2}$

$$
\begin{aligned}
& u^{(n)}(t)=r^{n}\left(\left\langle\begin{array}{c}
n \\
0
\end{array}\right\rangle\left(u-u_{1}\right)\left(u-u_{2}\right)^{n}+\left\langle\begin{array}{c}
n \\
1
\end{array}\right\rangle\left(u-u_{1}\right)^{2}\left(u-u_{2}\right)^{n-1}\right. \\
+ & \left.\left\langle\begin{array}{l}
n \\
2
\end{array}\right\rangle\left(u-u_{1}\right)^{3}\left(u-u_{2}\right)^{n-2}+\ldots+\left\langle\begin{array}{c}
n \\
n-1
\end{array}\right\rangle\left(u-u_{1}\right)^{n}\left(u-u_{2}\right)\right) \\
= & r^{n} \sum_{k=0}^{n-1}\left\langle\begin{array}{l}
n \\
k
\end{array}\right\rangle\left(u-u_{1}\right)^{k+1}\left(u-u_{2}\right)^{n-k}
\end{aligned}
$$

where $n=2,3, \ldots$.

The above formula (5) has been discussed during the Conference ICNAAM 2006 (September 2006) held in Greece and it appeared, with an inductive proof, in paper [6] (see also [7]). Independently, the formula has been considered and proved, with a proof based on generating functions, by Franssens [1].

The polynomial, of order $(n+1)$ of the variable $u$, appearing on the right hand side of equation (5) is known in the literature as the derivative polynomial. It can be proved (see [8]) that all $(n+1)$ roots of the polynomial are simple and lie in the interval $\left[u_{1}, u_{2}\right]$. The derivative polynomials were intensively studied recently.

\section{Further properties of the logistic function and its derivatives}

Formula (5) applied to the particular form (2) of the logistic equation is as follows:

$$
u^{(n)}(t)=\left(-\frac{c}{u_{\max }}\right)^{n} \cdot \sum_{k=0}^{n-1}\left\langle\begin{array}{l}
n \\
k
\end{array}\right) u^{k+1}\left(u-u_{\max }\right)^{n-k} .
$$

The polynomial of the variable $u$, of order $(n+1)$ on the right hand side of (6) is uniform in the sense of the following remark. 
Remark 1. If a number $u_{0}$ is the root of the polynomial on the right hand side of (6) i.e.,

$$
\sum_{k=0}^{n-1}\left\langle\begin{array}{l}
n \\
k
\end{array}\right\rangle u_{0}^{k+1}\left(u_{0}-u_{\max }\right)^{n-k}=0,
$$

then dividing both sides of (7) by $u_{\max }^{n+1}$ we get

$$
\sum_{k=0}^{n-1}\left\langle\begin{array}{l}
n \\
k
\end{array}\right\rangle\left(\frac{u_{0}}{u_{\max }}\right)^{k+1}\left(\frac{u_{0}}{u_{\max }}-1\right)^{n-k}=0 .
$$

Thus, $u_{0}$ is a root of the derivative polynomial on the right hand side of (6), if $u_{0} / u_{\max }$ is the root of the polynomial

$$
\begin{aligned}
& u^{\prime}(t)=u(1-u)=-u(u-1)=P_{2}(u), \\
& u^{\prime \prime}(t)=u(u-1)^{2}+u^{2}(u-1)=P_{3}(u), \\
& u^{\prime \prime \prime}(t)=-u(u-1)^{3}-4 u^{2}(u-1)^{2}-u^{3}(u-1)=P_{4}(u), \\
& u^{(4)}(t)=u(u-1)^{4}+11 u^{2}(u-1)^{3}+11 u^{3}(u-1)^{2}+u^{4}(u-1)=P_{5}(u), \\
& u^{(5)}(t)=-u(u-1)^{5}-26 u^{2}(u-1)^{4}-66 u^{3}(u-1)^{3}-26 u^{4}(u-1)^{2} \\
& -u^{5}(u-1)=P_{6}(u) .
\end{aligned}
$$

All roots of the polynomials $P_{k}(u),(k=3,4,5,6)$ can be calculated explicitely, so the polynomials can be factored and we get

$$
\begin{aligned}
& P_{3}(u)=2 u(u-1)\left(u-\frac{1}{2}\right), \\
& P_{4}(u)=-6 u(u-1)\left(u-\frac{1}{2}-\frac{\sqrt{3}}{6}\right)\left(u-\frac{1}{2}+\frac{\sqrt{3}}{6}\right), \\
& P_{5}(u)=24 u(u-1)\left(u-\frac{1}{2}\right)\left(u-\frac{1}{2}-\frac{\sqrt{6}}{6}\right)\left(u-\frac{1}{2}+\frac{\sqrt{6}}{6}\right), \\
& P_{6}(u)=-120 u(u-1)\left(u-\frac{1}{2}-\frac{\sqrt{30(15-\sqrt{105})}}{60}\right)\left(u-\frac{1}{2}-\frac{\sqrt{30(15+\sqrt{105})}}{60}\right) \\
& \left(u-\frac{1}{2}+\frac{\sqrt{30(15-\sqrt{105})}}{60}\right)\left(u-\frac{1}{2}+\frac{\sqrt{30(15+\sqrt{105})}}{60}\right) .
\end{aligned}
$$

Therefore, the least positive roots of the polynomials are

$$
\begin{aligned}
& P_{4}(u): \quad \frac{1}{2}-\frac{\sqrt{3}}{6} \approx 0,211, \\
& P_{5}(u): \quad \frac{1}{2}-\frac{\sqrt{6}}{6} \approx 0,0917, \\
& P_{6}(u): \quad \frac{1}{2}-\frac{\sqrt{30(15+\sqrt{105})}}{60} \approx 0,0413 .
\end{aligned}
$$

Let us write down, using formula (6) and the notation of (8) the first few derivatives of the logistic function, which fulfills equation (2).

By Remark 1 we can assume, without loss of the generality, that $u_{\max }=1$ and $c=1$.

We obtain successively: 
Thus, by using Remark 1 we see that, for example, if at some point of time $t_{0}$ (the least possible) $u^{\prime \prime \prime}\left(t_{0}\right)=0$ ( $u^{\prime \prime}\left(t_{0}\right)$ is a local maximum), then the value of the logistic function at this point is $u\left(t_{0}\right)=0,211 u_{\max }$.

In Fig. 7, we see two characteristic points of the exemplary logistic curve (with the same parameters as on Fig. 1) the inflection point (zero of the second derivative $\left.u^{\prime \prime}(t)\right)$ and the zero of the third derivative $u^{\prime \prime \prime}(t)$.
Similar conclusions can be drawn for the least zeroes of the $u^{(4)}(t)$ (the polynomial $P_{5}(u)$ is used in this case) or $u^{(5)}(t)\left(P_{6}(u)\right)$ with the constants given above.

In Fig. 2-6 below, we can see graphs of the derivative polynomials $P_{k}(u)$ for $k=2,3,4,5,6$ respectively, for $u \in[0,1]$. The polynomials are symmetric (even or odd) with respect to the point $u=1 / 2$.

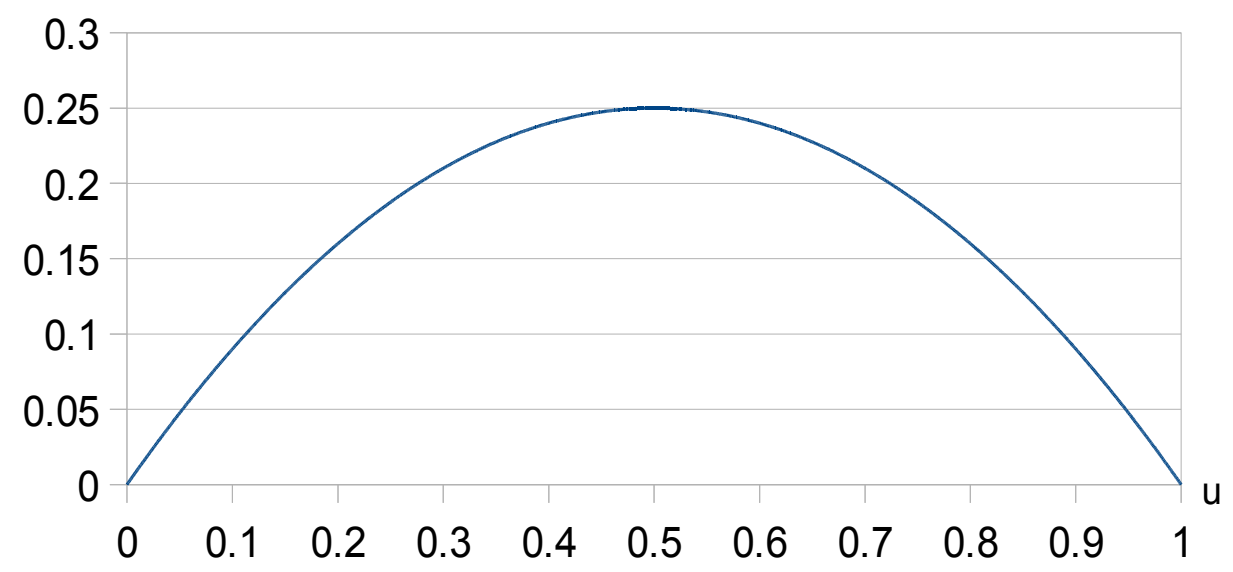

Figure 2. Polynomial $\mathrm{P}_{2}(\mathrm{u})$

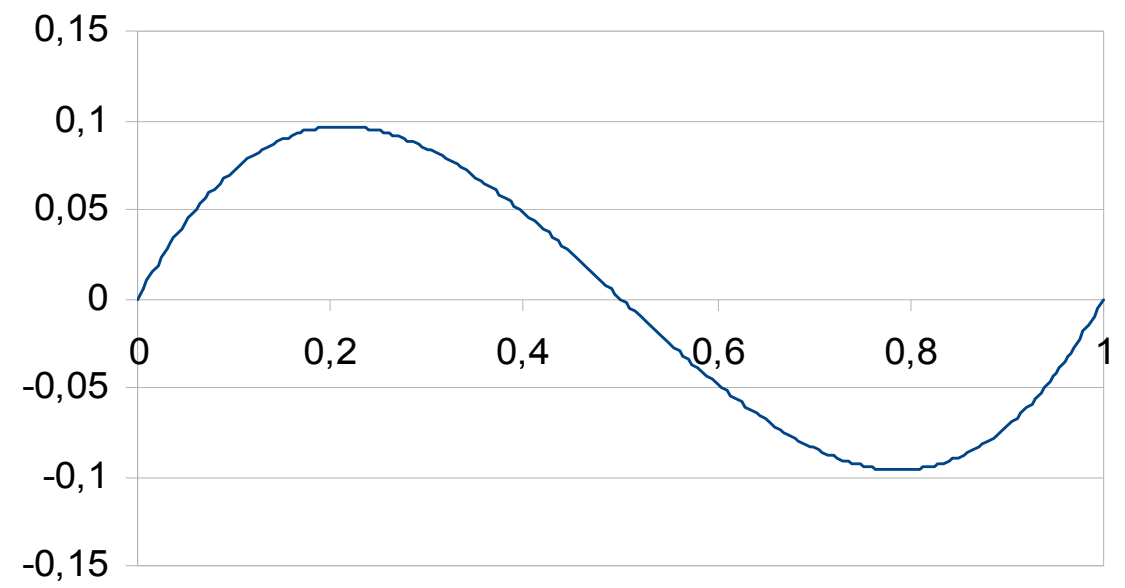

Figure 3. Polynomial $\mathrm{P}_{3}(\mathrm{u})$ 


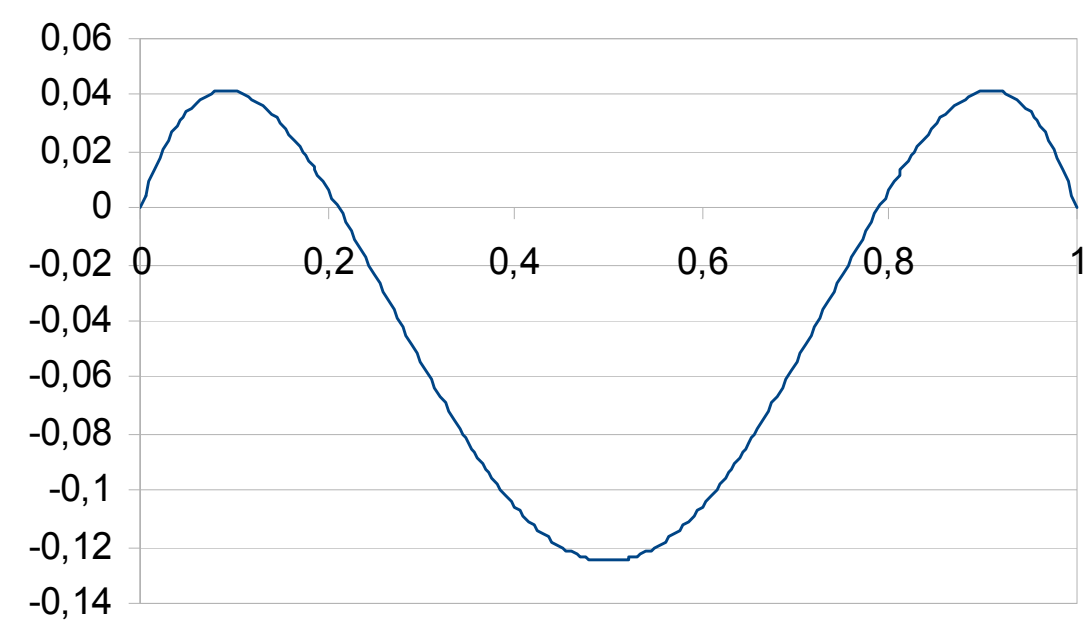

Figure 4. Polynomial $\mathrm{P}_{4}(\mathrm{u})$

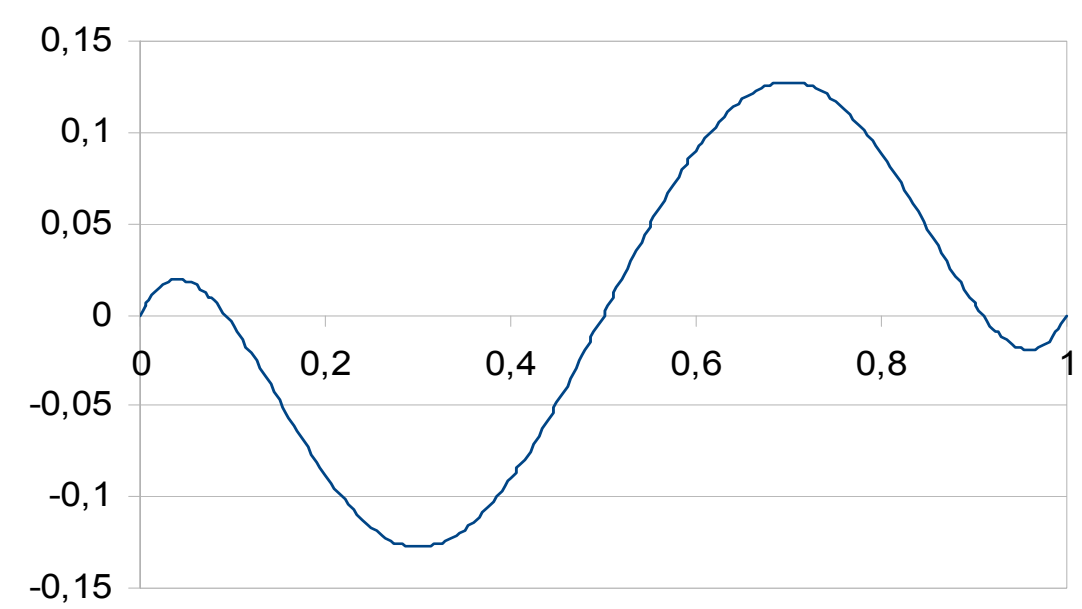

Figure. 5. Polynomial $\mathrm{P}_{5}(\mathrm{u})$

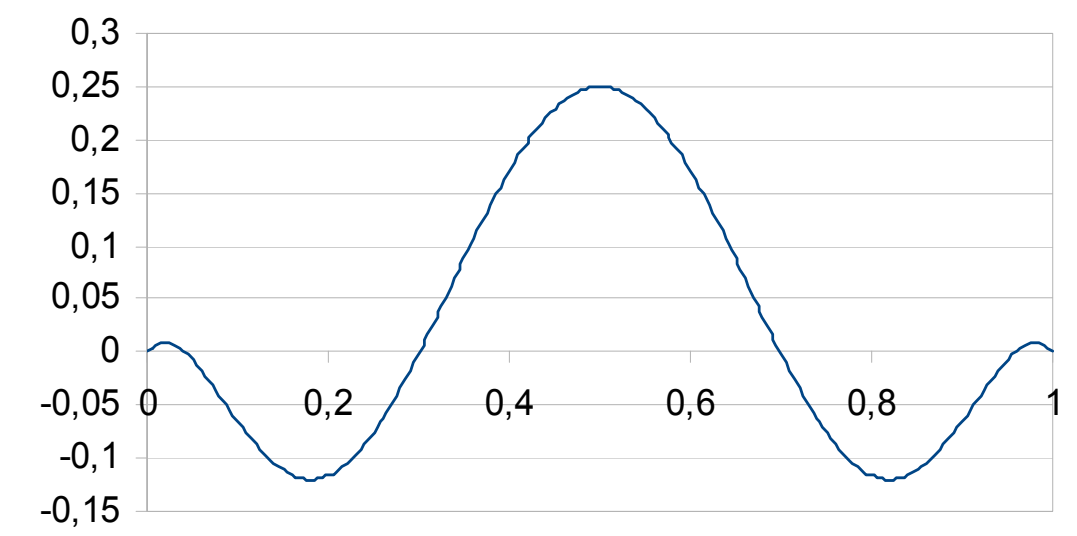

Figure 6. Polynomial $\mathrm{P}_{6}(\mathrm{u})$ 


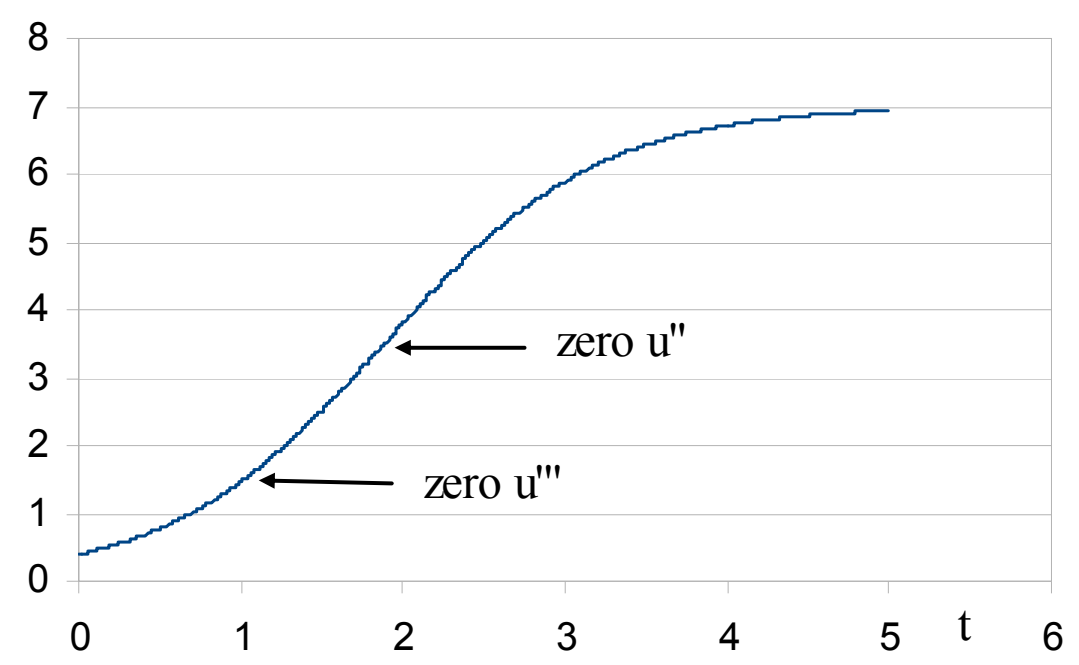

Figure 7. Two characteristic points of the logistic curve

\section{Some applications of the method}

\subsection{Loyalty cards in a chain of stores}

The data in the Table 2 represent the number of loyalty cards (NLC) issued in a large chain of stores in Poland. The observations relate to the period December 2011November 2013 (e.g., 47/12 means forty-seventh week of the year 2012).
In the initial period of time, covering the first 2 or 3 months, the phenomenon was a fairly rapid process, due to Christmas, New Year and a big promotional campaign. Then the situation stabilised and in the next weeks the total number of loyalty cards (Total NLC) proceeded according to a logistic curve. Fig. 8 shows the total number of issued cards starting from the tenth week of all observations.

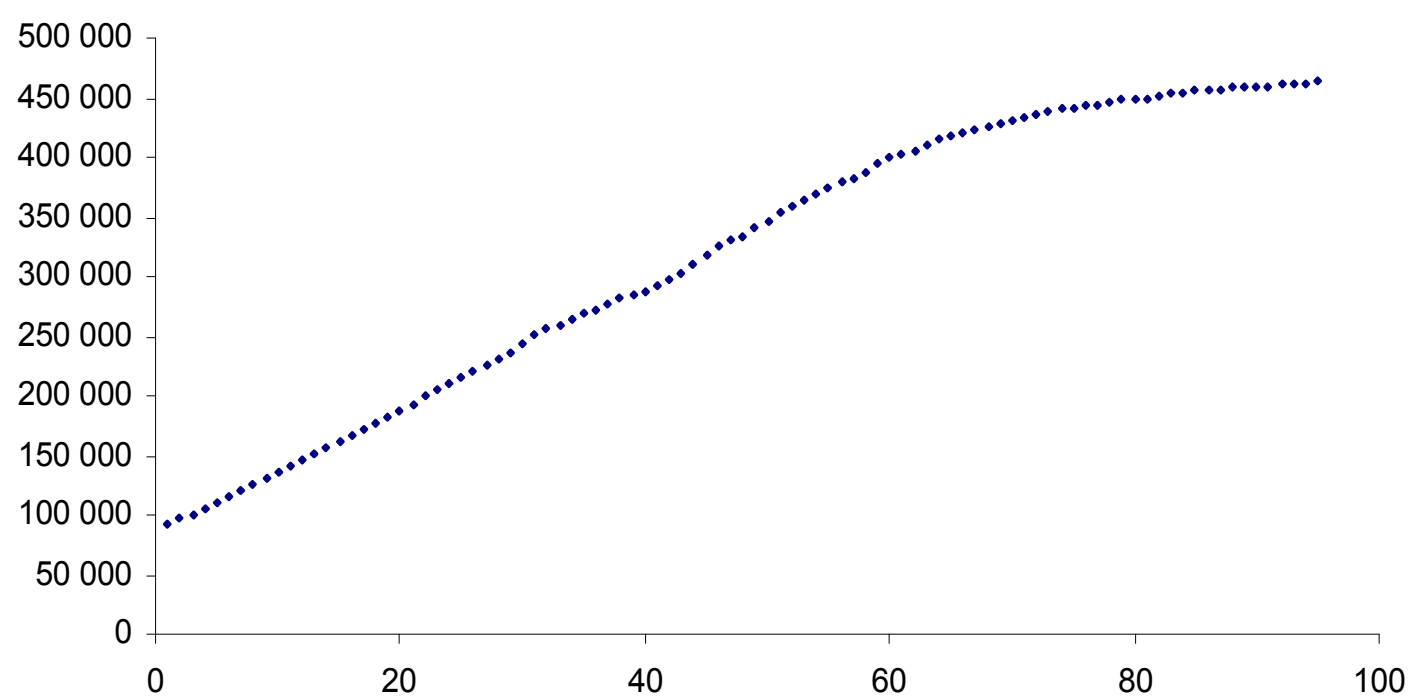

Figure 8 . The total number of loyalty cards issued (Total NLC) 
Table 2. The number of loyalty cards issued (NLC)

\begin{tabular}{|c|c|c|c|c|c|c|c|}
\hline Week & NLC & Week & NLC & Week & NLC & Week & NLC \\
\hline $48 / 11$ & 7236 & $23 / 12$ & 4307 & $50 / 12$ & 7741 & $25 / 13$ & 2077 \\
\hline $49 / 11$ & 11904 & $24 / 12$ & 5776 & $51 / 12$ & 8950 & $26 / 13$ & 1889 \\
\hline $50 / 11$ & 12887 & $25 / 12$ & 5561 & $52 / 12$ & 3447 & $27 / 13$ & 1686 \\
\hline $51 / 11$ & 10665 & $26 / 12$ & 5521 & $01 / 13$ & 3510 & $28 / 13$ & 1651 \\
\hline $52 / 11$ & 5616 & $27 / 12$ & 5525 & $02 / 13$ & 6334 & $29 / 13$ & 1402 \\
\hline $01 / 12$ & 7133 & $28 / 12$ & 5625 & $03 / 13$ & 6793 & $30 / 13$ & 1247 \\
\hline $02 / 12$ & 8428 & $29 / 12$ & 5393 & $04 / 13$ & 6846 & $31 / 13$ & 2026 \\
\hline $03 / 12$ & 7263 & $30 / 12$ & 5132 & $05 / 13$ & 5764 & $32 / 13$ & 1847 \\
\hline $04 / 12$ & 7135 & $31 / 12$ & 5768 & $06 / 13$ & 5803 & $33 / 13$ & 899 \\
\hline $05 / 12$ & 7038 & $32 / 12$ & 5826 & $07 / 13$ & 5121 & $34 / 13$ & 1132 \\
\hline $06 / 12$ & 6173 & $33 / 12$ & 4683 & $08 / 13$ & 4223 & $35 / 13$ & 1920 \\
\hline $07 / 12$ & 5061 & $34 / 12$ & 5337 & $09 / 13$ & 4955 & $36 / 13$ & 1551 \\
\hline $08 / 12$ & 4237 & $35 / 12$ & 7216 & $10 / 13$ & 3939 & $37 / 13$ & 1172 \\
\hline $09 / 12$ & 4953 & $36 / 12$ & 6396 & $11 / 13$ & 3566 & $38 / 13$ & 935 \\
\hline $10 / 12$ & 5536 & $37 / 12$ & 5325 & $12 / 13$ & 7844 & $39 / 13$ & 903 \\
\hline $11 / 12$ & 5387 & $38 / 12$ & 4421 & $13 / 13$ & 5085 & $40 / 13$ & 826 \\
\hline $12 / 12$ & 4868 & $39 / 12$ & 4111 & $14 / 13$ & 3158 & $41 / 13$ & 619 \\
\hline $13 / 12$ & 4673 & $40 / 12$ & 4343 & $15 / 13$ & 3550 & $42 / 13$ & 840 \\
\hline $14 / 12$ & 3496 & $41 / 12$ & 4462 & $16 / 13$ & 4468 & $43 / 13$ & 701 \\
\hline $15 / 12$ & 5474 & $42 / 12$ & 3780 & $17 / 13$ & 3498 & $44 / 13$ & 601 \\
\hline $16 / 12$ & 5576 & $43 / 12$ & 4048 & $18 / 13$ & 3726 & $45 / 13$ & 882 \\
\hline $17 / 12$ & 5245 & $44 / 12$ & 3708 & $19 / 13$ & 2339 & $46 / 13$ & 775 \\
\hline $18 / 12$ & 5196 & $45 / 12$ & 3474 & $20 / 13$ & 2628 & $47 / 13$ & 849 \\
\hline $19 / 12$ & 5563 & $46 / 12$ & 4462 & $21 / 13$ & 2708 & $48 / 13$ & 1238 \\
\hline $20 / 12$ & 5252 & $47 / 12$ & 3957 & $22 / 13$ & 3482 & & \\
\hline $21 / 12$ & 4616 & $48 / 12$ & 5405 & $23 / 13$ & 2142 & & \\
\hline $22 / 12$ & 5690 & $49 / 12$ & 7913 & $24 / 13$ & 2710 & & \\
\hline
\end{tabular}




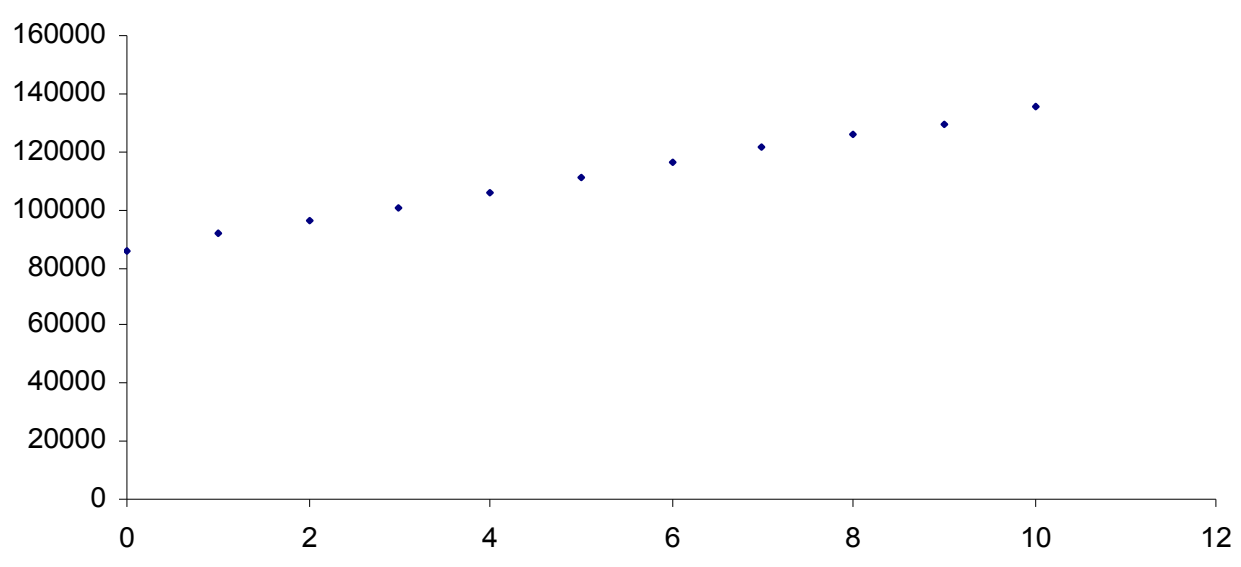

Figure 9. Initial data

We will try to use, to the above data, our theory described in the earlier sections. Let us take into account the first ten observations from the Fig. 8 (see the second and third columns of the Table 3 and Fig. 9). We calculate the successive differences for the data. By the second central difference (SCD) at time $t$ of an equally spaced time series $\left\{y_{t}\right\},(t=0,1,2,3, \ldots, n)$, we mean the number given by the formula

$$
\left(y_{t+1}-2 y_{t}+y_{t-1}\right) / 2 \text {. }
$$

The calculations are shown in Table 3. Instead of using SCD, we could use the second left differences (SLD) at time $t$ given by the formula:

$$
\left(y_{t}-2 y_{t-1}+y_{t-2}\right) / 2
$$

We see that the first local maximal value of the second central difference, 358, is taken for $t=3$ where the total number of issued loyalty cards is 100,776. Therefore, using comments from Section 3, we can estimate the saturation level as:

$$
u_{\text {max }}=100,776 / 0.211=477,611
$$

Because the value of SCD at point $t$ is equal to the value of SLD at $t+1$, then using in the decision the last one, leads to the estimation:

$$
u_{\max }=105,729 / 0.211=501,085
$$

Table 3. Second central differences (SCD) for the initial observations

\begin{tabular}{|c|c|c|c|}
\hline Week & No $(\boldsymbol{t})$ & Total NLC & SCD \\
\hline $05 / 12$ & 0 & 85305 & -556 \\
\hline $06 / 12$ & 1 & 91478 & -412 \\
\hline $07 / 12$ & 2 & 96539 & 358 \\
\hline $08 / 12$ & 3 & 100776 & 291.5 \\
\hline $09 / 12$ & 4 & 105729 & -74.5 \\
\hline $10 / 12$ & 5 & 111265 & -259.5 \\
\hline $11 / 12$ & 6 & 116652 & -9.5 \\
\hline $12 / 12$ & 7 & 121520 & -588.5 \\
\hline $13 / 12$ & 8 & 126193 & \\
\hline $14 / 12$ & 9 & 129689 & \\
\hline
\end{tabular}


Table 4. Diffusion of mobile telephony for Germany and Slovak Republic

\begin{tabular}{|c|c|c|c|c|}
\hline Year & Germany & SCD & Slovak Republic & SCD \\
\hline 1995 & 0.05 & & 0.01 & \\
\hline 1996 & 0.07 & 0.005 & 0.01 & 0.015 \\
\hline 1997 & 0.1 & 0.02 & 0.04 & 0.01 \\
\hline 1998 & 0.17 & 0.02 & 0.09 & -0.01 \\
\hline 1999 & 0.28 & 0.095 & 0.12 & 0.04 \\
\hline 2000 & 0.58 & -0.105 & 0.23 & 0.03 \\
\hline 2001 & 0.67 & -0.025 & 0.4 & -0.015 \\
\hline 2002 & 0.71 & 0.01 & 0.54 & 0 \\
\hline 2003 & 0.77 & 0.01 & 0.68 & -0.015 \\
\hline 2004 & 0.85 & 0.01 & 0.79 & -0.03 \\
\hline 2005 & 0.95 & -0.015 & 0.84 & 0.01 \\
\hline 2006 & 1.02 & 0.03 & 0.91 & 0.07 \\
\hline 2007 & 1.15 & -0.005 & 1.12 & -0.155 \\
\hline 2008 & 1.27 & -0.065 & 1.02 & 0.045 \\
\hline 2009 & 1.26 & -0.095 & 1.01 & 0.045 \\
\hline 2010 & 1.06 & 0.12 & 1.09 & -0.035 \\
\hline 2011 & 1.1 & -0.01 & 1.1 & 0.005 \\
\hline 2012 & 1.12 & & 1.12 & \\
\hline
\end{tabular}

Otherwise, we could find a polynomial, which best fits the data in the sense of the least square method and then investigate its second derivative for a maximum. Such polynomial, e.g. of order four, is as follows

$$
\begin{aligned}
& f(x)=-1.6807 x^{4}+30.515 x^{3} \\
& -206.23 x^{2}+5610.1 x+85584
\end{aligned}
$$

and its second derivative

$$
f^{\prime \prime}(x)=-20,16 x^{2}+183,09 x-412,46
$$

has a maximum at the point $x=4.5$. The value of $f(x)$ at this point is $f(4.5)=108,745$. Thus, we can estimate by starting a project to introduce a loyalty card, e.g. in a chain of stores, we could use the logistic curve to determine the increase in the number of loyal customers. At the same time, we could develop a promotion plan for the recruited persons, so that the budget of a given period of time (e.g., for a year) had a chance to be in a real way achieved.

During the project, the model created by the logistic curve allows us to monitor the effectiveness of the project, to draw conclusions and make appropriate decisions if there were derogations from our previous assumptions.

\subsection{Diffusion of mobile telephones in two countries}

Recently many papers have been published, which were devoted to mathematical modelling of the percentage diffusion over the population of mobile telephone for different countries (see [3, 5, 10 and 11]).

Consider two European countries, one with a stable and well-developed economy - Germany and the second, relatively recently accepted into the European Union - Slovak Republic, which transformed from a centrally planned to a market-driven economy. Table 4 shows the rate of mobile telephone subscriptions per one inhabitant in the two countries (see also Fig. 10 and 11). 


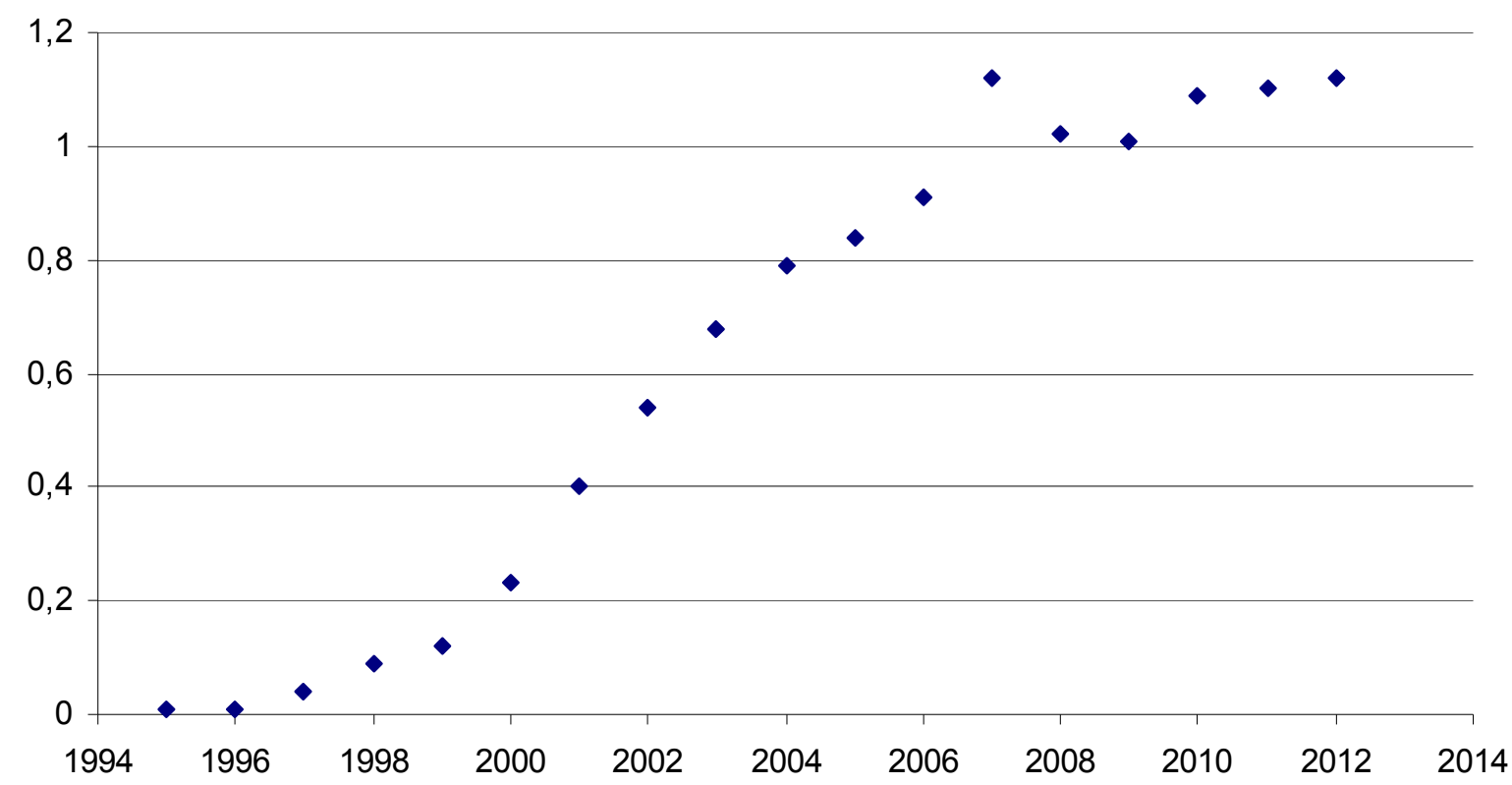

Figure 10. Rate of mobile telephone subscriptions per one inhabitant in Slovak Republic

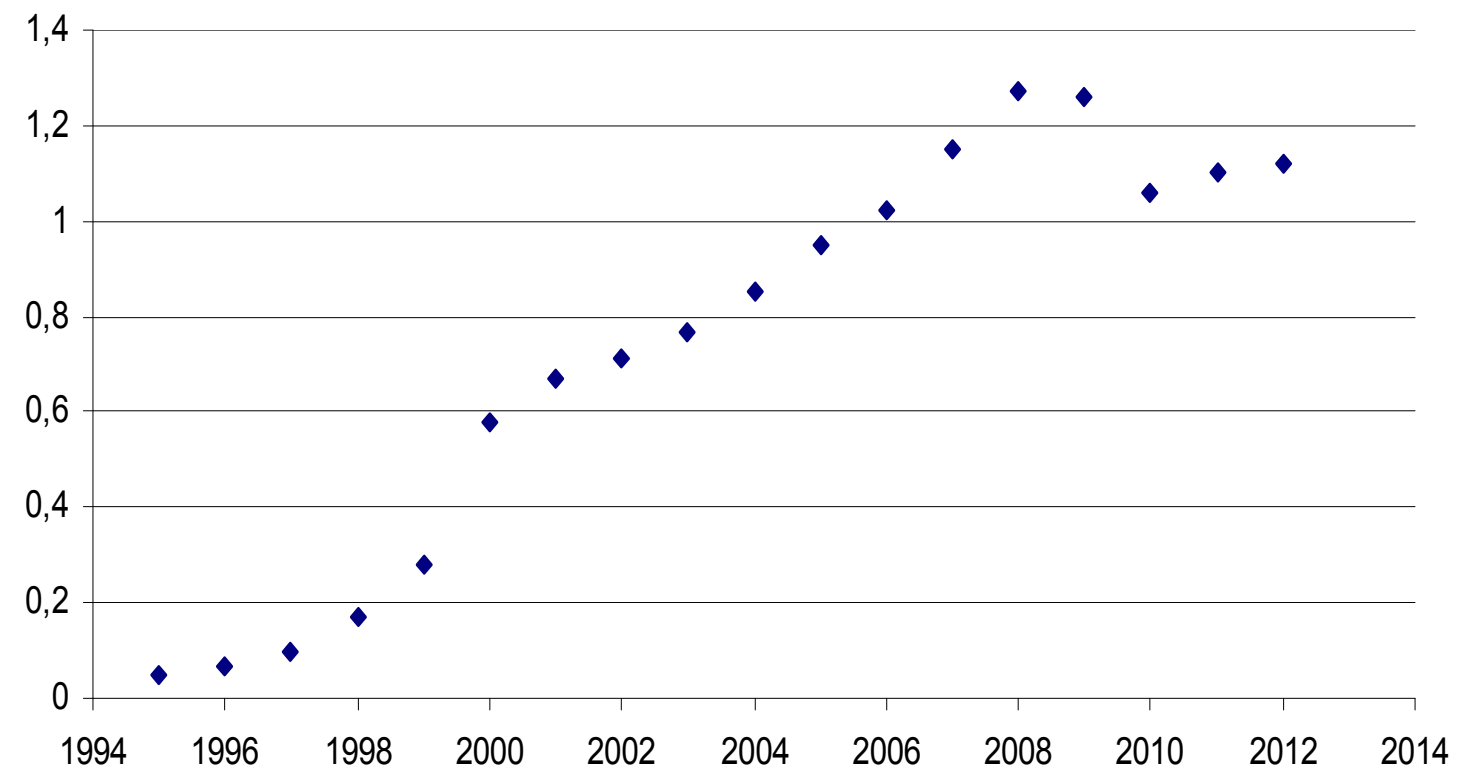

Figure 11. Rate of mobile telephone subscriptions per one inhabitant in Germany 
Table 5. The quantity of medical devices purchased (QMD)

\begin{tabular}{|c|c|c|c|c|c|c|c|}
\hline Month & QMD & Month & QMD & Month & QMD & Month & QMD \\
\hline $06 / 09$ & 3 & $03 / 10$ & 23 & $12 / 10$ & 15 & $09 / 11$ & 16 \\
\hline $07 / 09$ & 12 & $04 / 10$ & 8 & $01 / 11$ & 4 & $10 / 11$ & 17 \\
\hline $08 / 09$ & 8 & $05 / 10$ & 20 & $02 / 11$ & 11 & $11 / 11$ & 25 \\
\hline $09 / 09$ & 17 & $06 / 10$ & 11 & $03 / 11$ & 8 & $12 / 11$ & 12 \\
\hline $10 / 09$ & 22 & $07 / 10$ & 10 & $04 / 11$ & 8 & $01 / 12$ & 8 \\
\hline $11 / 09$ & 30 & $08 / 10$ & 15 & $05 / 11$ & 5 & $02 / 12$ & 5 \\
\hline $12 / 09$ & 15 & $09 / 10$ & 10 & $06 / 11$ & 9 & $03 / 12$ & 5 \\
\hline $01 / 10$ & 11 & $10 / 10$ & 17 & $07 / 11$ & 12 & & \\
\hline $02 / 10$ & 4 & $11 / 10$ & 16 & $08 / 11$ & 11 & & \\
\hline
\end{tabular}

The data, corresponding to the period from 2000 to 2012, were collected from the International Telecommunication Union (ITU, http://www.itu.int) and these corresponding to the period 1995-1999 were extracted from the paper C. Michalakelis, T. Sphicopoulos [5].

On the graph for Germany (see Fig. 11) are seen two perturbations and changes in the trend. They were probably caused by early 2000 s recession, which mainly occurred in developed countries and financial crisis of 2007-2008, which led to the 2008-2012 global recession. However in years 1995-2000, the shape well fits the logistic curve and we see from Table 4 that SCD clearly takes its maximal value at the point 0.28 .

Thus, the estimated saturation level is $u_{\max }=0.28 / 0.211$ $=1.327$. It seems interesting to note that, despite of the perturbations described above, a similar level has been reached in 2013, and was equal $1,301 .^{1}$

For fast-growing economy of Slovak Republic, the crises have not had much impact on the level of diffusion. Maximum of SCD, for the initial observations, is not as explicit as previously and should be fixed somewhere in the interval $[0.12,0.23]$. Therefore, the estimated level of saturation is from $0.12 / 0.211=$ 0.57 to $0.23 / 0.211=0.109$.

Let us note that SLD indicator would give us, in this case, the value of saturation level in the interval from $0.23 / 0.211=1.09$ to $0.4 / 0.211=1.89$.

\footnotetext{
1 'Research and Markets Adds Report: Germany - Telecoms, IP Networks and Digital Media'. TMC News, 12 June 2013. (retrieved 5.11.2013).
}

\subsection{Purchases of certain medical devices}

The data in Table 5 relate to some specific medical devices used in the diagnosis and treatment of patients. These products are used in public and private medical institutions.

The main users are public institutions, which buy these products using public funds in accordance with the 'Public Procurement Law'.

The average lifetime of the product is about 3 years. After this period, the device is subjected to a major renovation restoring its full functionality or is exchanged for a new one. The specificity of purchases from the budget indicates that purchases of these products are usually made in the fourth quarter of the calendar year. Increasing demand for these products contribute to periodic health programs implemented under

Fig. 12 shows the total quantity of purchases (on the horizontal axis the first month denotes 06/09 i.e., June 2009).

We see that only the initial data fit a logistic curve and then the phenomenon is no longer of such a nature. However, the maximum of SLD is reached in November 2009, where the total purchases are 92 devices. The estimated saturation level is

$$
u_{\max }=92 / 0.211=436
$$

and it seems to be a quite good forecast of its real value. 


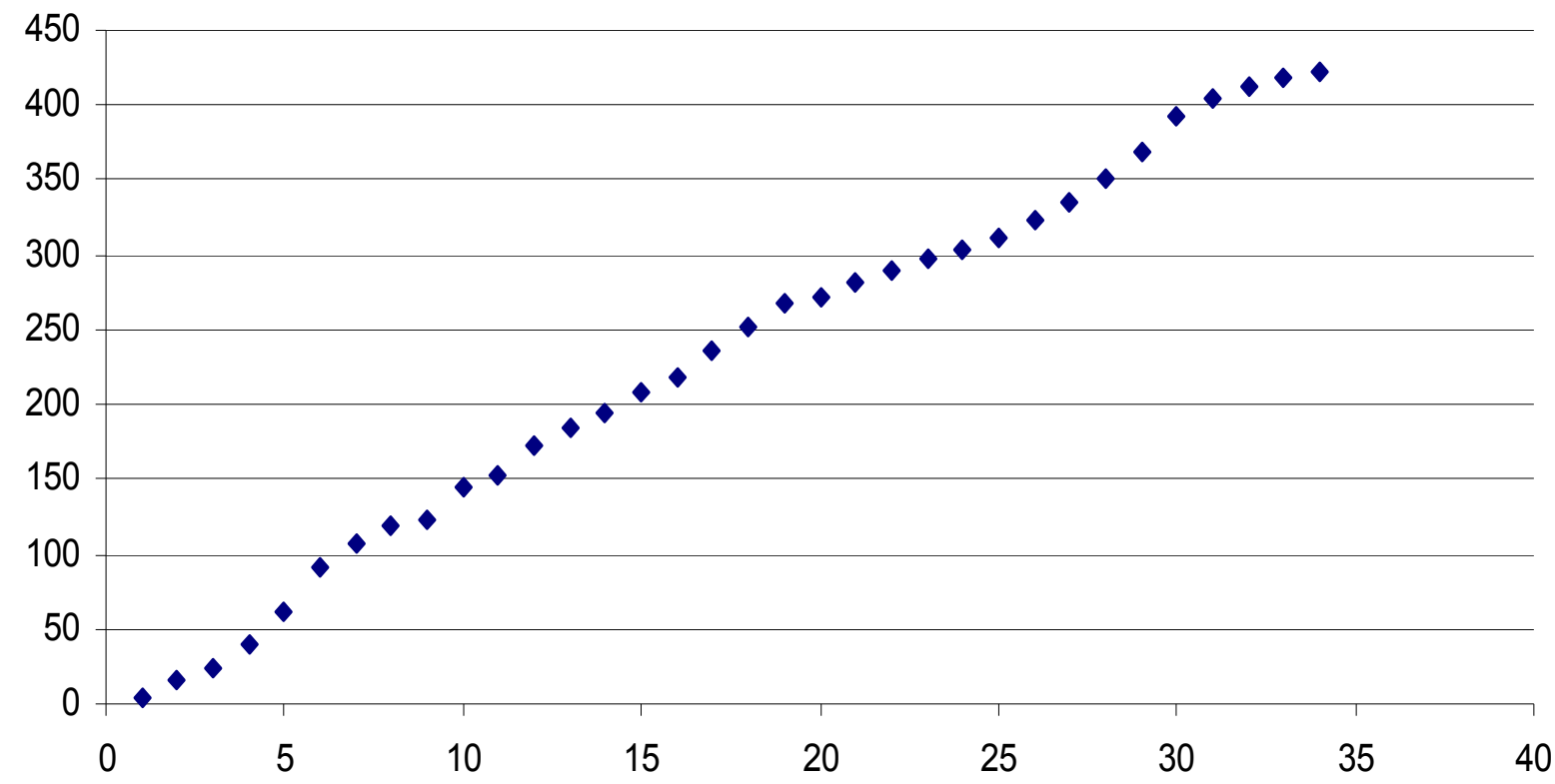

Figure 12. Total quantity of medical devices purchased

\section{Conclusions and future works}

In this paper, we have presented a method to study time series having the logistic trend. The approach is based on indicating some characteristic points corresponding to zeroes of successive derivatives of the logistic function in the data. We gave a general formula for the $n$th derivative of the logistic function, expressing it in terms of the derivative polynomials and Eulerian numbers. Then, we have computed first few derivatives and calculated zeroes of the corresponding derivative polynomials. It seems that from a practical point of view, especially with relatively small number of initial data, particularly significant is the leading zero of the third derivative, where the logistic function takes value $0.211 u_{\max }$.

We have shown the usefulness of our method with examples related to economics and management. We have demonstrated that when a phenomenon is at its early stages of development, then the saturation level may be effectively predicted.

We believe that this approach should be used together with existing methods, e.g. the nonlinear least squares method.

In our next research works, we will apply a similar idea for other mathematical models used in economics and management as Gompertz and Bass curves.

\section{Bibliography}

[1] Franssens G.R. - Functions with derivatives given by polynomials in the function itself or a related function [in] Analysis Mathematica 33(2007), pp. 17-36.

[2] Graham R.L., Knuth D.E., Patashnik O. - Concrete Mathematics: A Foundation for Computer Science, Reading MA: Addison Wesley, 1994.

[3] Junseok Hwang, Youngsang Cho, Nguyen Viet Long - Investigation of factors affecting the diffusion of mobile telephone services: An empirical analysis for Vietnam [in] Telecommunications Policy 33 (2009), pp. 534-543.

[4] Meade N., Islam T. - Modelling and forecasting the diffusion of innovation - A 25-year review [in] International Journal of Forecasting, 22 (2006), pp. 519-545.

[5] Michalakelis C., Sphicopoulos T. - A population dependent diffusion model with a stochastic extension [in] International Journal of Forecasting 28 (2012), pp. 587-606.

[6] Rządkowski G. - Eulerian numbers and Riccati's differential equation (eds. T.E. Simos) Proceedings of ICNAAM 2006, Wiley-VCH Verlag (2006), pp. 291-294.

[7] Rządkowski G. - Derivatives and Eulerian Numbers [in] Amer. Math. Monthly 115 (2008), pp. 458-460. 
[8] Rządkowski G. - On a family of polynomials [in] The Mathematical Gazette, 518 (2006), pp. 283286.

[9] Lixian Qian, Didier Soopramanien - Using diffusion models to forecast market size in emerging markets with applications to the Chinese car market [in]press Journal of Business Research, 2013.

[10] Feng-Shang Wu, Wen-Lin Chu - Diffusion models of mobile telephony [in] Journal of Business Research, 63 (2010), pp. 497-501.

[11] Yamakawa P., Rees G.H., Salas J.M., Alva N. The diffusion of mobile telephones: An empirical analysis for Peru [in] Telecommunications Policy, 37 (2013), pp. 594-606. 